\title{
Análise da avaliação da irregularidade longitudinal para aceitação de pavimentos de concreto
}

\begin{tabular}{|c|}
\hline MARCUS VINÍCIUS PAULA DE LIMA - ENGENHERO ME. \\
\hline $\begin{array}{l}\text { PALOMA MORAIS DE SOUZA - ENGENHEIRA } \\
\text { JOSÉ LEOMAR FERNANDES JÚNIOR - ENGENHEIRO DOUTOR }\end{array}$ \\
\hline Escola de ENGeENHaria de São Carlos da Universidade de São PAulo \\
\hline
\end{tabular}

\section{RESUMO}

MOTIVADO PELA IMPORTÂNCIA DA CONDIÇÃO INICIAL DE IRREGULARIDADE LONGITUDINAL NO DESEMPENHO DE PAVIMENTOS DE CONCRETO, ESTE ESTUDO ANALISA OS MÉTODOS DE AVALIAÇÃO E OS LIMITES NORMATIVOS UTILIZADOS NO BRAsIL PARA RECEBIMENTO E ACEITAÇÃO DE OBRAS, POR MEIO DE UM COMPARATIVO COM A REALIDADE DOS ESTADOS UNIDOS, REFERÊNCIA EM TERMOS DE NORMAS E MÉTOdOS EXECUTIVOS. PARA A ANÁLISE COMPARATIVA, REALIZOU-SE UM LEVANTAMENTO DAS ESPECIFICAÇÕES DE CADA ESTADO NORTE-AMERICANO E DAS NORMAS BRASILEIRAS. FOI OBSERVADO QUE A ADOÇÃO
DO ÍNDICE DE PERFIL COMO CRITÉRIO PARA AVALIAÇÃO DE IRREGULARIDADES EM PAVIMENTOS DE CONCRETO NO BRASIL VAI NO SENTIDO CONTRÁRIO AO MOVIMENTO DE PADRONIZAÇÃO DO IRI NOS ESTADOS NORTE-AMERICANOS. EM RELAÇÃO AOS LIMITES NORMATIVOS, OS VALORES PARA ACEITAÇÃO DOS PAVIMENTOS NOS ESTADOS NORTE-AMERICANOS SÃO MAIS RIGOROSOS QUE OS LIMITES BRASILEIROS, O QUE INFLUENCIA A QUALIDADE DE ROLAMENTO INICIAL E RESULTA EM UM MENOR CUSTO DE OPERAÇÃO DOS VEÍCULOS AO LONGO DO PERÍODO DE PROJETO.

Palavras-chave: irregularidade longitudinal, pavimentos de concreto, índice de perfil, gerência de pavimentos.

\section{INTRODUÇÃO}<smiles>C1C2CC12</smiles>

primeira estrada de pavimento em concreto no Brasil foi construída em 1925 entre Riacho Grande e Cubatão. Até o início da década de 1950, diversas obras foram realizadas com esse tipo de pavimento, porém, a partir da Segunda Guerra Mundial, a produção de cimento passou a ser direcionada para a indústria da construção civil, o que, juntamente com os preços baixos do asfalto na época, levaram a um direcionamento para a execução de pavimentos asfálticos.

A retomada na construção de pavimentos de concreto foi incentivada na década de 1990 pela aquisição de pavi- mentadoras de formas deslizantes e usinas dosadoras, o que representou um grande avanço em termos de produtividade na execução dos serviços. Junto a essa retomada, surgiu uma preocupação que não existia nas primeiras obras executadas até a década de 1950: a necessidade de avaliação de irregularidade longitudinal dos pavimentos.

No Brasil, a questão da irregularidade longitudinal inicial de pavimentos de concreto tem sido historicamente um problema para os empreiteiros, sendo prática comum a realização de correções de irregularidades em pavimentos novos com serviços de cepilhamento de camada, técnica que consiste no micronivelamento do pavimento com uma máquina de discos diamantados, criada para prolongar a vida útil do pavimento após anos de operação.

Neste cenário, este estudo objetiva realizar uma análise dos métodos empregados para avaliação da irregularidade longitudinal de pavimentos de concreto no Brasil e dos limites utilizados para sua aceitação. Para efeito comparativo, utilizou-se como base a realidade dos Estados Unidos, referência mundial em termos de normas e métodos executivos. Como análise complementar, é realizado um levantamento de boas práticas que podem ajudar a melhorar a condição inicial de superfície dos pavimentos de concreto brasileiros. 


\section{CRITÉRIOS PARA ACEITAÇÃO DA IRREGULARIDADE DOS PAVIMENTOS}

O levantamento realizado foi baseado nas especificações de serviços utilizadas nos Estados Unidos e no Brasil. Nessas especificações, prevalecem dois índices de avaliação de irregularidade: o International Roughness Index (IRI) e o Índice de Perfil (IP). O IRI tem como referência o modelo quarto-de-carro e o levantamento simula a resposta dinâmica de um veículo padrão ao trafegar sobre o perfil longitudinal da via. Já, o IP é uma medida de aceitação e controle da irregularidade obtida a partir dos excessos à blanking band (faixa de altura uniforme, posicionada entre os pontos de altos e baixos do perfil) no perfilograma.

\section{I Estados Unidos}

Nos Estados Unidos não existe uma norma que defina os procedimentos de avaliação e limites normativos para todo o país. Com isso, para a realização do levantamento foi necessária a verificação das especificações de serviço de cada um dos 50 departamentos de transportes estaduais. Na Figura 1, é apresentado o resultado da pesquisa sobre o índice definido por cada estado norte-americano para avaliação da irregularidade longitudinal.

Do total de cinquenta estados, vinte e nove utilizam o IRI como método padrão de avaliação de irregularidades e quinze adotam o IP. Entre os quinze estados que utilizam o IP, sete realizam o levantamento com blanking band nula (46,6\%), quatro com blanking band de $2,5 \mathrm{~mm}(26,7 \%)$ e quatro com blanking band de 5,0 mm (26,7\%). Os seis estados classificados como "outros" não

Tabela 1 - Limites normativos para pagamento integral da obra nos Estados Unidos

\begin{tabular}{|ccccc|}
\hline Índice & $\mathbb{P}_{0,0 \mathrm{~mm}}$ & $\mathbb{P}_{2,5 \mathrm{~mm}}$ & $\mathbb{P}_{5,0 \mathrm{~mm}}$ & $\mathbb{R}$ \\
\hline$N^{0}$ de estados & 7 & 4 & 4 & 29 \\
\hline $\begin{array}{c}\text { Menor valor limite* } \\
\text { (Estado) }\end{array}$ & 189,4 & 75,0 & 78,9 & 0,85 \\
(Connecticut) & (Arkansas) & (Flórida) & (Dakota do Norte) \\
\hline $\begin{array}{c}\text { Maior valor limite* } \\
\text { (Estado) }\end{array}$ & 631,3 & 252,5 & 157,8 & 1,56 \\
Média valores limites* & (lowa) & (Wyoming) & (Havaí) & (Nebraska) \\
\hline *Valores de IP em mm/km e de IRl em m/km. & 491,7 & 142,4 & 106,8 & 1,12 \\
\hline
\end{tabular}

possuem especificações para pavimentos de concreto ou utilizam um outro método de avaliação.

Em relação aos limites normativos, cada estado os define de forma independente. Com isso, mesmo entre aqueles que utilizam o mesmo índice e faixa de blanking band, os valores de irregularidade para aceitação da obra são diferentes. Na Tabela 1 são apresentados o menor e o maior limite normativo entre os estados que adotam o mesmo padrão de avaliação de irregularidade para pagamento integral da obra, além da média dos va-

lores limites.

Observa-se, na Tabela 1, que a média dos valores normativos para pagamento integral da obra entre os estados que utilizam o IP com blanking band nula é consideravelmente maior que a média daqueles que utilizam o IP com faixa de $2,5 \mathrm{~mm}$ e $5,0 \mathrm{~mm}$. Isso acontece devido à capacidade do levantamento com blanking band nula capturar desvios adicionais importantes para a determinação do nível de irregularidade longitudinal, considerando no cálculo do IP a superfície real do pavimento.

Por essa razão, índices determinados com faixas diferentes de blanking band não são comparáveis. A comparação, no entanto, pode ser realizada entre estados que utilizam o mesmo índice e faixa de blanking band, de maneira que se pode afirmar, por exemplo, que o valor normativo para aceitação 
Tabela 2 - Limite normativo e método de avaliação de irregularidade longitudinal no Brasil

\begin{tabular}{|c|c|c|c|c|}
\hline Órgão & Norma & Índice & Equipamento recomendado & Irregularidade limite \\
\hline DNIT & 049/2013 & $\mathbb{P}$ & Perfilógrafo Califórnia & $240 \mathrm{~mm} / \mathrm{km}^{\star}$ \\
\hline DER/SP & ET-P00/039 & $\mathbb{P}$ & Perfilógrafo Califórnia & 157,8 mm/km \\
\hline DER/PR & ES-P 35/05 & $|\mathrm{R}|$ & Tipo resposta ou perfilômetro & $2,5 \mathrm{~m} / \mathrm{km}$ \\
\hline
\end{tabular}

de irregularidade de pavimentos no estado do Havaí é mais flexível que o da Flórida, já que o limite de aceitação no primeiro estado é de $157,8 \mathrm{~mm} / \mathrm{km}$, enquanto no segundo é de $78,9 \mathrm{~mm} / \mathrm{km}$, ambos para 5,0 $\mathrm{mm}$ de blanking band.

\subsection{Brasil}

A especificação de serviço que regulamenta a execução de pavimentos de concreto com formas deslizantes no Brasil é a DNIT 049/2013, que indica, para a determinação da irregularidade longitudinal, o Perfilógrafo Califórnia e determina o IP médio de 240 mm/km como o limite máximo aceitável. No seu texto não é especificada a blanking band que deve ser utilizada nos levantamentos, tornando a informação sobre

o limite de aceitação incompleta, visto que o valor do IP varia de acordo com a faixa de supressão utilizada. Na prática, os levantamentos têm sido realizados com blanking band de 5,0 $\mathrm{mm}$.

Na Tabela 2, são apresentados os métodos de avaliação e os limites normativos do DNIT, do DER/SP e do DER/PR para aceitação de pavimentos quanto à irregularidade longitudinal.

Em relação aos valores normativos, nota-se que os limites nos Estados Unidos para aceitação do pavimento quanto à irregularidade são bem mais rigorosos que os exigidos pela norma DNIT 049/2013, com índices mais baixos mesmo nos estados com regulamentações mais flexíveis (que aceitam valores de $157,8 \mathrm{~mm} / \mathrm{km}$ para o $\mathrm{IP}_{5,0 \mathrm{~mm}}$ e $1,58 \mathrm{~m} / \mathrm{km}$ para o $\mid \mathrm{RI})$.
Lee (2007) re-

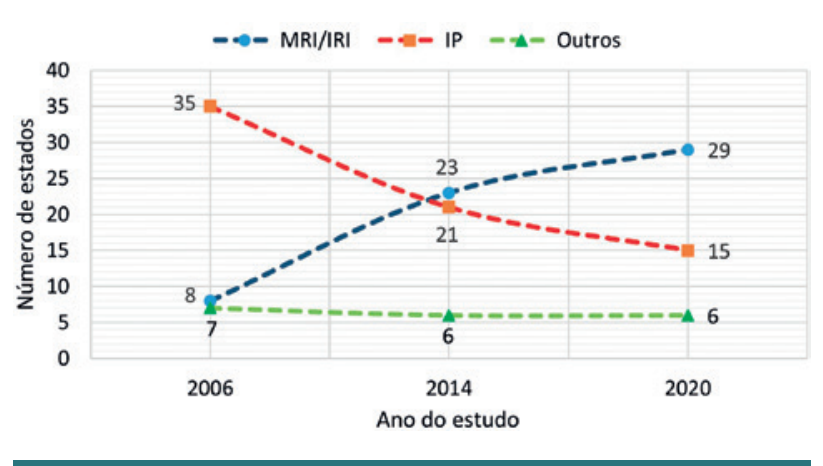

\section{Figura 2}

Número de estados que adotam o IP e o IRI para avaliar pavimentos de concreto

Fonte: Lima (2020) iniciais elevados de irregularidade levam a maiores custos de reabilitação e a uma menor vida útil do pavimento. No caso específico dos pavimentos de concreto de cimento Portland, dimensionados
20 anos de vida de serviço, uma elevada irregularidade longitudinal inicial é extremamente indesejável, pois levará à um mau desempenho do pavimento a longo prazo.

\subsection{Análise dos critérios $d \epsilon$ avaliação utilizados}

Pesquisas semelhantes sobre os métodos de avaliação de irregularidade longitudinal nos estados norte-americanos foram realizadas por Wilde (2007), baseado nas especificações estaduais vigentes em 2006, e por Merritt, Chang e Rutledge (2015), com dados de 2014. Na Figura 2 são apresentadas as mudanças no decorrer do tempo em termos de número total de estados que adotam cada índice de avaliação de irregularidade longitudinal (IP e IRI).

Fica evidente o número crescente de estados que passaram a adotar o IR| para avaliação da irregularidade longitudinal em pavimentos de concreto nos Estados Unidos. Entre 2006 e 2020, o uso do IRI cresceu $42 \%$, tornando-se o método padrão de avaliação em 58\% dos estados norte-americanos. Enquanto isso, o IP que antes era utilizado por $70 \%$ dos estados, passou a ser o método padrão em apenas 30\% deles.

Há várias razões para a prepondepara, no mínimo, rância do IRI em relação ao IP, começando pela velocidade dos levantamentos, pois os perfilômetros não exigem a interrupção do tráfego e podem ser realizados a velocidades muito maiores do que a dos perfilógrafos ( $5 \mathrm{~km} / \mathrm{h}$ ), que ainda requerem o fechamento das pistas (WILDE, 2007). Outro motivo é a incapacidade dos levantamentos com perfilógrafos de medir comprimentos de onda superiores ao seu vão (7,62 m), ao passo que o IRI também é influenciado 
pelos comprimentos de onda maiores, resultando em uma melhor representação dos perfis longitudinais.

Além disso, como o IRI é o índice utilizado em programas computacionais de gerência de pavimentos para a programação de manutenção e reabilitação dos pavimentos, informações do seu valor logo após a construção são fundamentais para uma boa simulação da propagação de defeitos. Nesse caso, conversões equitativas de IP para $|R|$ não são recomendadas por dependerem de fatores locais, como tipo de pavimento, clima e das características dos equipamentos de medição utilizados.

No Brasil, a utilização da blanking band de 5,0 mm para determinação do IP restringe a sensibilidade do levantamento a grandes picos individuais, não sendo uma medida representativa dos desvios totais do pavimento, conforme comprovado por Wilde (2007). Assim como vem ocorrendo nos Estados Unidos, a tendência no Brasil é da adoção dos perfilômetros inerciais como método padrão de levantamento da irregularidade longitudinal dos pavimentos de concreto, tendo o IRI como a propriedade quantificadora, o que adequaria a realidade brasileira aos avanços ocorridos nas últimas décadas em países desenvolvidos.

\section{BOAS PRÁTICAS PARA A MELHORIA DA IRREGULARIDADE LONGITUDINAL INICIAL}

$\mathrm{Na}$ sequência é apresentada a estratégia de incentivo/desincentivo utilizada por grande parte dos estados norte-americanos e que impulsionou os empreiteiros a buscarem formas de melhorar os níveis de irregularidades de pavimentos de concreto nos Estados
Unidos. Além disso, é apresentada uma das soluções encontradas, o stringless pavement, que começou a ser utilizado no Brasil a partir de 2017.

\section{I Práticas de incentivo/ desincentivo aos empreiteiros nos Estados Unidos}

Para obter menores índices de irregularidade inicial em suas obras, a maioria dos departamentos estaduais dos Estados Unidos possuem políticas de incentivo/desincentivo aos empreiteiros baseadas nos níveis de irregularidade longitudinal do pavimento construído. A política consiste em oferecer bonificação financeira as construtoras que entregarem pavimentos com índices de irregularidade abaixo dos limites normatizados e penalizá-las com descontos em medição, caso os valores sejam superiores aos permitidos. Na Figura 3, é possível observar a política de pagamento adotada por cada estado norte-americano.

De acordo com Merritt, Chang e Rutledge (2015), políticas de incentivo aos empreiteiros se justificam, pois, o número reduzido de manutenções e a vida útil mais longa resultante dos pavimentos de melhor qualidade compensam o pagamento extra realizado. Da mesma forma, caso os empreiteiros não cumpram os requisitos básicos, os fundos de desincentivo retidos do pagamento dos serviços servirão para ajudar a custear os gastos resultantes da vida útil mais curta do pavimento e do aumento de manutenção.

Evans, Darter e Egan (2005) relataram a experiência do estado do Tennessee com incentivos aos empreiteiros. Segundo os autores, os construtores atingiram em média bonificação de 6\% dos valores de contrato, com os resultados obtidos, e as melhorias em relação aos limites normativos aumentaram em 14\% a vida útil dos trechos construídos.

No Brasil, o exigido quanto a irregularidade longitudinal é a entrega dos segmentos com índices abaixo do limite normativo, não havendo nenhuma especificação baseada no desempenho. A exceção é o estado de São Paulo, que adota a política de desincentivo para índices superiores aos limites estabelecidos, porém sem o pagamento de incentivos. Dessa forma, do ponto de vista prático, os 
construtores que entregam pavimentos com irregularidades bem abaixo das especificadas em norma recebem a mesma contrapartida do governo daqueles que executam com irregularidades próximas ao limite normativo.

Ao exemplo dos Estados Unidos, os órgãos reguladores brasileiros poderiam adotar práticas de incentivo aos construtores como forma de encorajá-los a investir em equipamentos e tecnologias para melhorar os índices iniciais de irregularidade dos pavimentos construídos. Como consequência, os pavimentos nacionais teriam maior vida útil e os custos operacionais dos veículos e os gastos com manutenção das vias seriam reduzidos.

\subsection{Stringless pavement}

Um exemplo de investimento para promover a melhoria dos índices iniciais de irregularidade dos pavimentos é a utilização da tecnologia 3D para guiar pavimentadoras de concreto de formas deslizantes, conhecida internacionalmente como Stringless Pavement. O sistema consiste na execução de pavimentos de concreto sem a utilização das tradicionais linhas guias, responsáveis pelo direcionamento e altura da máquina, eliminando os efeitos da mudança de temperatura e umidade no tensionamento das linhas guias e a formação de catenárias, problemas que impactam diretamente os níveis de irregularidade do pavimento.

No Stringless Pavement, um conjunto de estações totais robóticas (ETR) fornece dados de posicionamento da pavimentadora em tempo real, que são comparados ao projeto embarcado no seu computador de

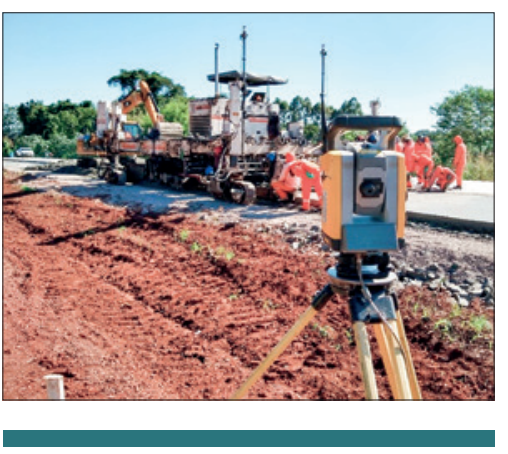

\section{Figura 4}

Execução de pavimento de concreto de cimento Portland na BR-163/PR com a tecnologia do stringless pavement

Fonte: Lima (2020)

bordo, para fornecer os comandos de acionamento hidráulico. Com acurácia de $\pm 3 \mathrm{~mm}$, essa tecnologia é utilizada com sucesso há mais de uma década em países da Europa e nos EUA.

No Brasil, até o momento, apenas uma pavimentadora de concreto trabalha guiada pelo sistema 3D, com operação iniciada em fevereiro de 2017 em um trecho da BR163 nas proximidades da cidade de Cascavel/PR. No ano seguinte, a máquina trabalhou na duplicação da BR-163/364/MT e, somados os trechos executados nas duas obras, estima-se que 160 km já foram pavimentados com o sistema. A Figura 4 mostra a pavimentadora trabalhando guiada pela tecnologia na obra de duplicação da BR-163/PR.

Com relação a capacidade da tecnologia3D em entregar pavimentos com índices de irregularidades bem abaixo dos limites normativos atuais, as primeiras experiências no Brasil são animadoras. $\mathrm{Na}$ análise dos resultados de IP em 59 segmentos da BR-163/364/MT construídos com o Stringless Pavement, mais de 95\% tiveram valores abaixo de 120 mm/ $\mathrm{km}$ e nenhum deles superou o valor normativo de $240 \mathrm{~mm} / \mathrm{km}$.

A necessidade de realização de investimentos na modernização e adaptação das pavimentadoras de concreto, e no treinamento dos principais envolvidos com o sistema, representam razões pelo qual o Stringless Pavement ainda não se tornou mais difundido no Brasil. A maioria desses investimentos, no entanto, pode ser recuperada com as vantagens propiciadas pela própria tecnologia, devendo o gestor de obras analisar o custo-benefício que a implantação do sistema pode trazer para a realidade da sua empresa.

\section{CONCLUSÕES}

Ao comparar os índices utilizados para avaliação de irregularidade de pavimentos de concreto de cimento Portland no Brasil e nos Estados Unidos, pode-se concluir que a recomendação para utilização do IP pela norma DNIT 049/2013 segue em sentido oposto à adoção do $|\mathrm{R}|$ pelos estados norte-americanos, método este que possui maior velocidade na realização dos levantamentos, melhor representação do perfil longitudinal e possibilidade de aplicação direta em programas computacionais de gerência de pavimentos.

Em relação aos limites normativos, observa-se um maior rigor das especificações norte-americanas para a aceitação de pavimentos novos quanto à irregularidade longitudinal. A adoção pelas normas brasileiras de critérios mais rigorosos quanto à irregularidade longitudinal 
dos pavimentos no início da vida em serviço pode trazer benefícios aos usuários e aumentar a vida útil do pavimento, reduzindo os gastos com manutenção e reabilitação.

Complementarmente, poderiam ser adotadas especificações por desempenho, com bônus e penalizações fixados com base no valor inicial da irregularidade longitudinal dos pavimentos. Uma consequência natural dessa medida seria o investimento em equipamentos para execução e controle de qualidade em obras, como o uso do sistema 3D em pavimentadoras de concreto de formas deslizantes, tecnologia que já vem sendo utilizada no Brasil e que proporciona redução considerável na irregularidade longitudinal dos pavimentos.

\section{AGRADECIMENTOS}

Os autores agradecem à Coordenação de Aperfeiçoamento de Pessoal de Nível Superior - Brasil (CAPES) pelas bolsas de estudo recebidas.

\section{DREFERÊNCIAS BIBLIOGRÁFICAS}

[1] EVANS, Lynn D.; DARTER, Michael I.; EGAN, Brian K. Development and Implementation of a Performance-Related Specification for I-65 Tennessee. United States. Federal Highway Administration, 2005.

[2] LEE, D. G. Dynamic Prediction Model of As-Built Roughness in Asphaltic Concrete Pavement Construction. Journal of Transportation Engineering, Vol. 133, No. 2, February 1, 2007. CASCE, ISSN 1943-5436, D0I: 10.1061/(ASCE)0733-947X(2007)133:2(90).

[3] LIMA, M. V. P. Tecnologia 3D em pavimentadoras de concreto de formas deslizantes: efeitos no processo executivo e na qualidade e desempenho do pavimento. 2020. Dissertação de mestrado. Universidade de São Paulo.

[4] MERRITT, D. K.; CHANG, G.K.; RUTLEDGE; J. L. 2015. Best Practices for Achieving and Measuring Pavement Smoothness, a Synthesis of State-of-Practice. FHWA LA.14/550, 2015. Louisiana Transportation Research Center, Baton Rouge, LA.

[5] WILDE, W. James. Implementation of an international roughness index for Mn/DOT pavement construction and rehabilitation. Technical Report No. MN/RC-200709. Minnesota Department of Transportation. EUA. 2007.

\section{KIT de PRÁTICAS RECOMENDADAS sobre ENSAIOS de DURABILIDADE das ESTRUTURAS de CONCRETO}

O conjunto de Práticas Recomendadas Sobre os Ensaios de Durabilidade das Estruturas de Concreto é fruto do trabalho do Comitê Técnico IBRACON/ALCONPAT 702 Procedimentos para Ensaios de Avaliação da Durabilidade das Estruturas de Concreto.
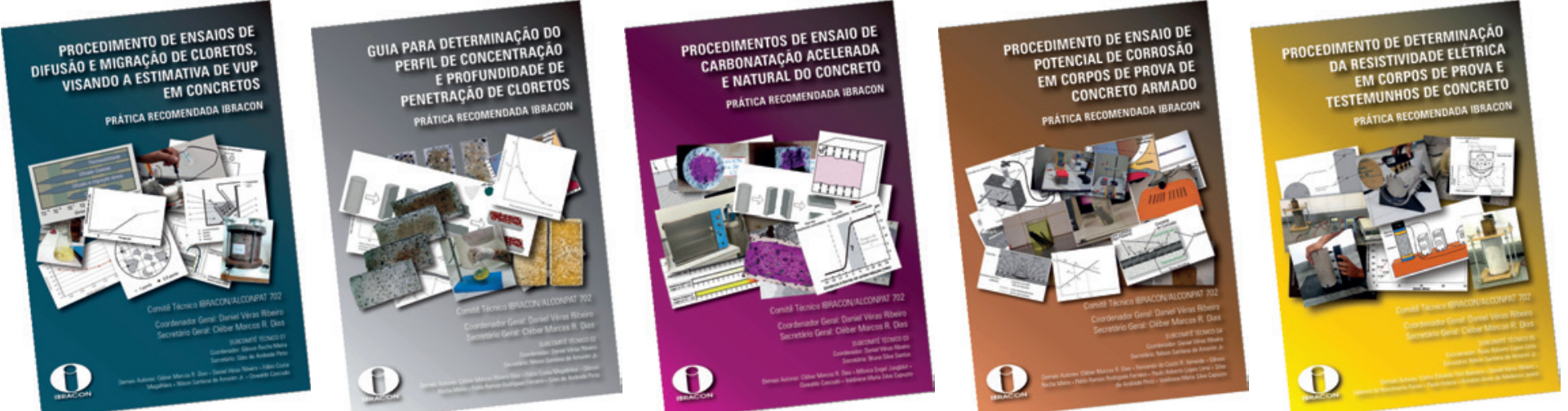

PROMOÇÃO: Kit com 5 Práticas + Guia de Prevenção da Reação Álcali-Agregado SÓCIOS: R\$ 300,00 | NÃO SÓCIOS: R\$ 550,00 Adquira o seu na Loja Virtual do IBRACON: http//lojaibracon.org.br

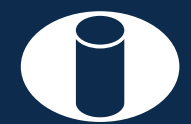

www.ibracon.org.br

f $\mathrm{i}$ ibraconOffice office@ibracon.org.br 